\section{Corneal sensitivity and subjective complaints of ocular pain in patients with fibromyalgia}

\author{
Abstract \\ Purpose Fibromyalgia (FM) is a chronic pain \\ disorder associated with pain and \\ hypersensitivity in various parts of the body. \\ The aim of this study was to understand \\ whether changes in corneal sensitivity were \\ associated with the subjective complaints of \\ these patients. \\ Patients and methods In this study, we \\ included 36 patients with FM (30 female, 6 \\ male, mean age: $46.7 \pm 9.2$ years) and 39 \\ healthy control subjects ( 33 females, \\ 6 males, mean age: $44.3 \pm 7.6$ years). We \\ performed a detailed ophthalmological \\ examination, measured tear film breakup \\ time (TBUT), and performed Schirmer \\ I test without anesthetic (SIT). Only \\ patients that did not have any eye \\ disease were included in this study. \\ Subjective complaints of the patients \\ were evaluated with ocular surface disease \\ index (OSDI) scores and the corneal sensation \\ was evaluated with Cochet-Bonnet \\ esthesiometer. \\ Results There was no significant difference \\ between the groups for age, sex, and visual \\ acuity. Both SIT (16.1 mm vs $15.3 \mathrm{~mm}$, \\ $P=0.36)$ and TBUT results (17.8 s vs $18.8 \mathrm{~s}$, \\ $P=0.40$ ) were similar in FM group and the \\ controls subjects. However, corneal sensations \\ in central $(60.0 \mathrm{~mm}$ vs $55.0 \mathrm{~mm}, P=0.03)$, \\ superior $(57.5 \mathrm{~mm}$ vs $50.0 \mathrm{~mm}, P=0.005)$, and \\ inferior $(53.89 \mathrm{~mm}$ vs $46.03 \mathrm{~mm}, P<0.001$ ) \\ regions were significantly increased in FM \\ patients. There was a significant positive \\ correlation between corneal sensation and \\ OSDI scores. \\ Conclusion In this study, we have \\ demonstrated that the patients with FM have \\ increased corneal sensitivity and have related \\ ocular surface complaints similar to dry eye \\ disease in the absence of it. \\ Eye (2018) 32, 763-767; doi:10.1038/eye.2017.275; \\ published online 26 January 2018
}

V Aykut ${ }^{1}$, A Elbay², I Çigdem Uçar ${ }^{3}$, F Esen ${ }^{1}$, A Durmus $^{3}, \mathrm{R} \mathrm{Karadag}^{1}$ and $\mathrm{H} \mathrm{Oguz}^{1}$

\section{Introduction}

Fibromyalgia syndrome (FM) is a chronic pain disorder that is characterized with generalized musculoskeletal pain, fatigue, cognitive dysfunction, sleep disturbances, and mood disorders. The etiology of FM is not completely known but neurosensory sensitization is a generally accepted mechanism in the pathogenesis. Several studies reported the prevalence of FM as high as $4.9 \%$ in females and $2.9 \%$ in males. ${ }^{1-5}$ Neurosensory sensitization may affect perception of pain in various medical conditions. LluchGirbes et al ${ }^{6}$ found that patients who have osteoarthritis with sensitization had perceived more pain than those without. In a meta-analysis, Fingleton et $a l^{7}$ found that patients who had osteoarthritis with sensitization had more severe symptoms compared with patients without sensitization. Similarly, previous studies on FM have shown that FM is associated with various neurobiological and psychological factors that can induce chronic central pain. Unlike the other senses (touch, hearing, smell, and taste), adaptation cannot develop in pain because of wind-up phenomenon. Repeated application of constant low intensity pain stimulus amplifies pain, only as a feature of pain. This pain amplification leads to increased sensation of pain. This amplification mechanism is controlled by supraspinal centers and it is impaired in FM. $^{8}$ Consequently, a painful stimulus with low amplitude is perceived as intense pain. ${ }^{9,10}$

In clinical practice, sometimes FM patients present to ophthalmology clinics with complaints of subjective ocular pain in the absence of any ocular disease. In general, clinicians prefer to prescribe these patients lubricants, as if they had dry eye disease. ${ }^{11}$ Most of these patients can not benefit from this treatment and keep visiting other
${ }^{1}$ Department of Ophthalmology, Goztepe Education and Research Hospital, Istanbul Medeniyet University School of Medicine, Istanbul, Turkey

${ }^{2}$ Department of Ophthalmology, Bezmialem University School of Medicine, Istanbul, Turkey

${ }^{3}$ Department of Ophthalmology, Istanbul Medeniyet University Goztepe Education and Research Hospital, Istanbul, Turkey

Correspondence: $\checkmark$ Aykut, Istanbul Medeniyet University, Goztepe Ĕgitim ve Araştırma Hastanesi, Goz Hastaliklari Klinigi, Kadikoy, Istanbul 34730, Turkey Tel: +90 505 4534570; Fax: +90 2165666614 . E-mail: v-aykut@hotmail. com

Received: 5 September 2017 Accepted in revised form: 22 October 2017 Published online: 26 January 2018 
ophthalmologists with similar complaints and still do not get any improvement in their condition.

The Cochet-Bonnet test is a subjective test that is used to evaluate corneal sensation. In this test, a nylon monofilament is touched to the corneal surface and perceived length of the filament is recorded. The CochetBonnet test is preferred for the evaluation of corneal sensitivity after corneal surgeries or in various ocular surface diseases. ${ }^{12,13}$

In this study, we have evaluated subjective complaints of FM patients with ocular surface disease index (OSDI) and studied corneal sensation with Cochet-Bonnet test in FM patients. Our aim in this study was to understand whether the subjective complaints of FM patients in the absence of ocular pathology or dry eye disease were related with a central change in the perception of corneal sensation.

\section{Materials and methods}

FM patients followed at physical therapy and rehabilitation clinic of our hospital were referred to our ophthalmology clinic. An age and gender matched control group was selected among ophthalmology clinic patients, who applied to our clinic for routine eye health control. Ethical approval for carrying out this clinical study was obtained from the Istanbul Medeniyet University institutional review board on human research. The study was conducted in accordance with the tenets of the Declaration of Helsinki. Informed consent was obtained from all participants upon explanation of the purpose and procedure of the study.

A detailed ophthalmic and medical history was obtained and OSDI scores were recorded in all cases. All participants underwent a comprehensive ophthalmic examination including measurement of best corrected visual acuity (BCVA), slit lamp examination, indirect ophthalmoscopy, tonometry, tear film breakup time (TBUT), Schirmer I test without anesthetic (SIT), and Cochet-Bonnet esthesiometer test. All of FM patients fulfilled American College of Rheumatology 2010 diagnostic criteria, which includes the following: (1) to have a widespread pain index score (indicative of the number of body regions that test positive for pain; range, $0-19$ ) of $\geq 7$; (2) to have a symptom severity scale score (a systematic measurement of secondary symptoms present in FM; range, $0-12$ ) of $\geq 5$; (3) to have had persistent symptoms for at least 3 months; and (4) to not have an overlapping disorder that might otherwise explain these symptoms. ${ }^{1}$

Subjects with dry eye diseases, blepharitis, conjunctivitis, keratitis, uveitis, glaucoma, or other disorders, which may lead to ocular pain, patients with allergic, inflammatory, or infectious diseases at the orbital or periorbital area, patients with headache caused by diseases other than FM were excluded from the study. Subjects who had a history of diseases that might cause a loss in corneal sensation such as herpetic keratitis, trachoma, Stevens Johnson syndrome, or participants with a history of a ophthalmic surgery were excluded from the study.

\section{Tear film breakup time}

A fluorescein strip was placed into the inferior conjunctival fornix for staining and removed. The time interval between the last complete blink and the appearance of the first dry spot or discontinuity on the tear film was measured with a stopwatch, in order to evaluate tear film stability and quality. TBUT $<10 \mathrm{~s}$ was considered abnormal.

\section{Schirmer I test}

SIT was performed without topical anesthesia in order to measure tear production. A $5 \times 35 \mathrm{~mm}$ filter paper strip was placed on the lateral one third of the inferior fornix without touching cornea. Five minutes after insertion, the length of the strip wetted by tears was recorded in millimeters.

\section{Cochet-Bonnet esthesiometer test}

Cochet-Bonnet esthesiometer consists of a nylon filament $60 \mathrm{~mm}$ in length and $0.12 \mathrm{~mm}$ in diameter. After the patient's head was placed on chinrest of the slit lamp, the filament was brought to contact with corneal epithelium very smoothly at a perpendicular angle until a slight bending of the filament is observed. Starting from $60 \mathrm{~mm}$ different filament lengths with $5 \mathrm{~mm}$ interval was tried until the patient noticed the smooth contact on their cornea. Measurements were repeated in five different corneal locations (central, superior, inferior, nasal, and temporal). All of the dry eye tests and corneal sensation tests were performed by the same clinician (ICU) for every patient.

Statistical analyses were performed using the SPSS software (ver. 20.0; SPSS Inc, Chicago, IL, USA). The distribution pattern of the data was analyzed with Kolmogorov-Smirnov test. Normally distributed data was presented as mean \pm SD and non-normally distributed data was presented as median (minimummaximum). Comparisons between groups with normally distributed data were done with Student $t$-test and MannWhitney $U$-test was used preferred in case of non normal distribution. Correlation statistics were performed with Pearson or Spearman correlation analysis, according to 
the distribution of the data. $P$-values below 0.05 were accepted as statistically significant.

\section{Results}

Thirty-six patients with FM (30 females, 6 males, mean age $46.7 \pm 9.2$ years) and 39 healthy individuals (33 females, 6 males, mean age $44.3 \pm 7.6$ years) were included in this case-control study. There was no statistically significant difference between the groups for age, sex, BCVA, SIT, and TBUT results (Table 1). OSDI scores were significantly higher in the patients with FM $(19.89 \pm 13.70)$ compared with healthy controls $(6.13 \pm 4.09)(P<0.001)$.

Corneal sensation was $60.0 \mathrm{~mm}$ (min. 45.0-max. 60.0) at the central cornea, $57.5 \mathrm{~mm}$ (min. 45.0-max. 60.0) superiorly, $53.89 \pm 5.23 \mathrm{~mm}$ inferiorly, $55.00 \pm 4.86 \mathrm{~mm}$ temporally, and $54.44 \pm 5.14 \mathrm{~mm}$ nasally in the FM group. In the control group, corneal sensation was $55.0 \mathrm{~mm}$ (min. 45.0-max. 60.0) at the central cornea, $50.0 \mathrm{~mm}$ (min. 35.0max. 60.0) superiorly, $46.03 \pm 6.71 \mathrm{~mm}$ inferiorly, $53.85 \pm 4.36$ temporally, and $53.72 \pm 4.83 \mathrm{~mm}$ nasally. The corneal sensation results at the central, superior, and inferior regions were significantly higher in the FM group compared with control group $(P=0.033, P=0.005$, and $P<0.001$, respectively). However, there was no statistically significant difference at temporal and nasal regions between the groups $(P=0.282$ and $P=0.518$, respectively) (Table 2 ).

We have also studied whether there was a correlation with dry eye related findings and corneal sensation in these patients (Table 3 ). There was a significant positive correlation between corneal sensation results from all five regions of cornea and OSDI scores seperately. However, we did not observe any correlation between corneal sensation results and SIT or TBUT results.

\section{Discussion}

There are two sensitization mechanisms: central sensitization and peripheral sensitization. FM is generally accepted as a condition associated with central sensitization. Peripheral sensitization is also suggested to contribute pathogenesis of FM. In central mechanism, inhibitory neural signals that descend from the supraspinal centers to the brainstem can be reduced or impaired. ${ }^{9-10}$

The normal function of inhibitory neural signals is restriction of pain. Central sensitivity syndrome may occur together with peripheral sensitization or may occur afterwards. C and A $\delta$ fibers are thinly myelinated, slightly larger, and highly selective and higher threshold nociceptors, whereas $\mathrm{A} \beta$ fibers are larger myelinated,
Table 1 Demographic characteristics of fibromyalgia patients and control subjects

\begin{tabular}{lccr}
\hline & Study & Control & P-values \\
\hline Participant $(n)$ & 36 & 39 & \\
Gender (m/f) & $30 / 6$ & $33 / 6$ & $0.879^{\mathrm{a}}$ \\
Age (years) & $46.72 \pm 9.18$ & $44.23 \pm 7.65$ & $0.204^{\mathrm{b}}$ \\
Schirmer (mm/5 min) & $16.11 \pm 3.96$ & $15.31 \pm 3.70$ & $0.367^{\mathrm{b}}$ \\
TBUT (s) & $17.81 \pm 5.60$ & $18.87 \pm 5.37$ & $0.403^{\mathrm{b}}$ \\
OSDI & $19.89 \pm 13.70$ & $6.13 \pm 4.09$ & $<0.001^{\mathrm{b}}$ \\
BCVA (logMAR) & $0.0(0.0-0.2)$ & $0.0(0.0-0.2)$ & $0.486^{\mathrm{c}}$ \\
\hline
\end{tabular}

Abbreviations: BCVA, best corrected visual acuity; $\mathrm{m} / \mathrm{f}$, male/female; TBUT, tear break up time. ${ }^{\mathrm{a}} \chi^{2}$-test. ${ }^{\mathrm{b}}$ Student's $t$-test. ${ }^{\mathrm{c}}$ Mann-Whitney $U$-test.

highly specialized, and have low-stimulus threshold mechanoreceptors that senses touch stimuli. Long-term and intense painful stimulus leads to genotypic and structural changes on the $\mathrm{A} \beta$ fibers and their mechanoreceptors. These changes can lead to peripheral sensitization. Therefore, $A \beta$ fibers transmit their touch signals as pain.

These changes also occur in upper neural centers (dorsal horn of the spinal cord and the trigeminal sensory nucleus). Postsynaptic receptors are continually stimulated by proinflammatory mediators, which are released from activated microglia in the synapse region, even when peripheral afferent stimuli are ceased. Thus, central sensitization can also arise from peripheral sensitization. ${ }^{14-19}$

Studies on patients with osteoarthritis revealed that sensitized patients perceive pain more severely than others. ${ }^{6,7}$ In a functional magnetic resonance imaging study, Gracely et $a l^{20}$ showed that the amplitude of pressure stimulus required to activate in cerebral pain-processing areas of the brain is lower in FM patients compared with healthy individuals. Biochemical data on FM also supports the central sensitization hypothesis. In these studies, the amount of substance $\mathrm{P}$, which is a pronociceptive substance, was found higher whereas the antinociceptive noradrenaline metabolite (3-methoxy-4-hydroxyphenylene) was in lower concentration in FM patients. These peptides have an important role in pain processing. Currently, many drugs that are developed for FM treatment show their effects by increasing the decreased adrenergic activity in the central nervous system. ${ }^{21-22}$

In ophthalmology practice, we encounter central sensitization in patients who have sensitized trigeminal brainstem nuclear complex, which perceives corneal sense. These patients report a different stinging sensation in their eyes. In this condition, different stimuli arising from cornea is perceived as pain (allodynia) or lowdensity painful stimuli is perceived too strongly (hyperalgesia). ${ }^{23-26}$ 
Table 2 Corneal sensations at five points in fibromyalgia patients and control group

\begin{tabular}{lccr}
\hline & Study & Control & P-values \\
\hline Central (min. -max.) & $60.0(45.0-60.0)$ & $55.0(45.0-60.0)$ & $0.033^{\mathrm{a}}$ \\
Superior (min. -max.) & $57.5(45.0-60.0)$ & $50.0(35.0-60.0)$ & $0.005^{\mathrm{a}}$ \\
Inferior (mean \pm SD) & $53.89 \pm 5.23$ & $46.03 \pm 6.71$ & $<0.001^{\mathrm{b}}$ \\
Temporal (mean \pm SD) & $55.00 \pm 4.86$ & $53.85 \pm 4.36$ & $0.282^{\mathrm{b}}$ \\
Nasal (mean \pm SD) & $54.44 \pm 5.14$ & $53.72 \pm 4.83$ & $0.518^{\mathrm{b}}$ \\
\hline
\end{tabular}

${ }^{\mathrm{a}}$ Mann-Whitney U-test. ${ }^{\mathrm{b}}$ Independent samples T-test.

Table 3 Correlations between corneal sensation results and dry eye disease parameters

\begin{tabular}{lccccc}
\hline & Central $^{\mathrm{a}}$ & Superior $^{\mathrm{a}}$ & Inferior $^{\mathrm{b}}$ & Temporal $^{\mathrm{b}}$ & Nasal $^{\mathrm{a}}$ \\
\hline Schirmer & $r=0.271$ & $r=0.279$ & $r=0.241$ & $r=-0.301$ & $r=0.207$ \\
& $P=0.11$ & $P=0.10$ & $P=0.157$ & $P=0.075$ & $P=0.226$ \\
TBUT & $r=0.241$ & $r=-0.099$ & $r=0.197$ & $r=0.202$ & $r=-0.016$ \\
& $P=0.157$ & $P=0.565$ & $P=0.249$ & $P=0.237$ & $P=0.928$ \\
OSDI & $r=0.441$ & $r=0.432$ & $P=0.338$ & $r=0.379$ & $r=0.468$ \\
& $P=0.007$ & $P=0.009$ & $P=0.022$ & $P=0.004$ \\
\hline
\end{tabular}

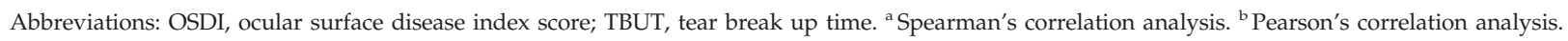

Shtein et $a^{23}$ demonstrated that central corneal sensitivity was higher in dry eye disease and FM patients, whereas they found that corneal nerve fiber density had only decreased in confocal microscopy measurements of the FM group, but not in the dry eye disease group. The Schirmer test results and TBUT scores were also lower in FM and dry eye groups compared with control group in that study. There was no correlation between corneal nerve density and corneal sensitivity, but the reason for this was not explained in the study. ${ }^{23}$ Similarly, other confocal microscopy studies also confirmed that FM patients had a thinner and less dense subbasal nerve plexus structure. ${ }^{24-25}$ Gallar et al ${ }^{26}$ compared $20 \mathrm{FM}$ patients and 18 healthy subjects, to evaluate corneal sensation in FM. They found that corneal sensitivity to chemical stimulation, heat and cold was increased, while sensitivity to mechanical stimulation remained unchanged. The authors attributed this change to dry eye disease in these patients, as FM group had lower Schirmer test score. ${ }^{26}$ In these studies, patients with FM were not separated from dry eye disease patients and even reported that many FM patients had findings related with dry eye disease.

Therefore, it was not possible to predict whether corneal sensitivity was affected by dry eye disease or FM in those studies.

In this study, we excluded patients with SIT score of $<10 \mathrm{~mm} / 5 \mathrm{~min}$ and a TBUT of $<10 \mathrm{~s}$. Thus, we were able to prevent the impact of dry eye disease on OSDI scores in our study. We measured corneal sensation at five distinct areas and different results were obtained. Sensitivity was significantly higher in the upper, lower, and central areas of the cornea in FM patients compared with control subjects. These findings were consistent with the abovementioned mechanisms. It is also possible that the impact of FM on corneal subbasal nerve plexus may not be uniform in different sections of the cornea.

Dry eye disease is one of the most common ocular pathologies affecting around $20 \%$ of adults older than 45 years. ${ }^{27}$ We also know that, many patients who are referred with severe dry eye complaints, have exaggerated responses to environmental stimuli or tear drops, which does not normally cause irritation or are mildly irritating. There are also many studies that report neurosensory dysfunction such as a symptom of dry eye disease. In routine clinical practice, many clinicians perceive or even treat this condition such as dry eye disease. ${ }^{11}$

Here we observed that patients with FM had increased OSDI scores in the absence of dry eye disease. In order to exclude the possibility that these patients might suffer a subclinical reduction in tear production or quality, we performed Schirmer test and TBUT. Our results indicated that there was no statistically significant difference in tear quantity or quality between our FM group and control subjects. Although SIT and TBUT scores did not correlate with corneal sensation, OSDI scores correlated significantly with all corneal sensation results from five different corneal regions we studied. Therefore, we believe that this increase in OSDI scores is related with the changes in corneal sensation rather than factors related with ocular surface pathologies. 


\section{Summary}

What was known before

- Fibromyalgia patients have increased prevalence of dry eye disease and complaints of ocular pain.

- Corneal sensation is increased in patients with fibromyalgia, most of whom have coexisting dry eye diasease.

What this study adds

- There are increased complaints of ocular pain in the absence of organic pathology in patients with fibromyalgia.

- Corneal sensitivity is increased in patients with fibromyalgia even in the absence of dry eye disease.

- The subjective complaints of ocular pain in fibromyalgia patients may arise from changes in central sensitisation and associated corneal sensitivity.

\section{Conflict of interest}

The authors declare no conflict of interest.

\section{References}

1 Wolfe F, Clauw DJ, Fitzcharles MA, Goldenberg DL, Katz RS, Mease P et al. The American College of Rheumatology preliminary diagnostic criteria for fibromyalgia and measurement of symptom severity. Arthritis Care Res 2010; 62(5): 600-610.

2 Gran JT. The epidemiology of chronic generalized musculoskeletal pain. Best Pract Res Clin Rheumatol 2003; 17(4): 547-561.

3 Branco JC, Bannwarth B, Failde I, Abello Carbonell J, Blotman F, Spaeth M et al. Prevalence of fibromyalgia: a survey in five European countries. Semin Arthritis Rheum 2010; 39(6): 448-453.

4 McGillion MH, Watt-Watson J, Stevens B, Lefort SM, Coyte P, Graham A. Randomized controlled trial of a psychoeducation program for the self-management of chronic cardiac pain. J Pain Symptom Manage 2008; 36(2): 126-140.

5 White KP, Speechley M, Harth M, Ostbye T. The London Fibromyalgia Epidemiology Study: the prevalence of fibromyalgia syndrome in London, Ontario. J Rheumatol 1999; 26(7): 1570-1576.

6 LluchGirbes E, Duenas L, Barbero M, Falla D, Baert IA, Meeus $\mathrm{M}$ et al. Expanded distribution of pain as a sign of central sensitization in individuals with symptomatic knee osteoarthritis. Phys Ther 2016; 96(8): 1196-1207.

7 Fingleton C, Smart K, Moloney N, Fullen BM, Doody C. Pain sensitization in people with knee osteoarthritis: a systematic review and meta-analysis. Osteoarthritis Cartilage 2015; 23(7): 1043-1056.

8 Pergolizzi J, Ahlbeck K, Aldington D, Alon E, Coluzzi F, Dahan A et al. The development of chronic pain: physiological CHANGE necessitates a multidisciplinary approach to treatment. Curr Med Res Opin 2013; 29(9): 1127-1135.

9 Lautenbacher S, Rollman GB. Possible deficiencies of pain modulation in fibromyalgia. Clin J Pain 1997; 13: 189-196.
10 Kosek E, Ekholm J. Modulation of pressure pain thresholds during and following isometric contraction. Pain 1995; 61: 481-486.

11 Rosenthal P, Baran I, Jacobs DS. Corneal pain without stain: is it real? Ocul Surf 2009; 7(1): 28-40.

12 Spierer O, Felix ER, McClellan AL, Parel JM, Gonzalez A, Feuer WJ et al. Corneal mechanical thresholds negatively associate with dry eye and ocular pain symptoms. Invest Ophthalmol Vis Sci 2016; 57(2): 617-625.

13 Bragheeth MA, Dua HS. Corneal sensation after myopic and hyperopic LASIK: clinical and confocal microscopic study. Br J Ophthalmol 2005; 89(5): 580-585.

14 Ji RR, Woolf CJ. Neuronal plasticity and signal transduction in nociceptive neurons: implications for the initiation and maintenance of pathological pain. Neurobiol Dis 2001; 8: 1-10.

15 Akopian AN, Sivilotti L, Wood JN. A tetrodotoxin-resistant voltage- gated sodium channel expressed by sensory neurons. Nature 1996; 379: 257-262.

16 Woolf CJ. Evidence for a central component of post-injury pain hypersensitivity. Nature 1983; 306: 686-688.

17 Hains BC, Saab CY, Klein JP, Craner MJ, Waxman SG. Altered sodium channel expression in second-order spinal sensory neurons contributes to pain after peripheral nerve injury. J Neurosci 2004; 24: 4832-4839.

18 Benoist JM, Gautron M, Guilbaud G. Experimental model of trigeminal pain in the rat by constriction of one infraorbital nerve: changes in neuronal activities in the somatosensory cortices corresponding to the infraorbital nerve. Exp Brain Res 1999; 126: 383-398.

19 Flor H. Cortical reorganisation and chronic pain: implications for rehabilitation. J Rehabil Med 2003; (41 Suppl): 66-72.

20 Gracely RH, Petzke F, Wolf J, Clauw DJ. Functional MRI evidence of augmented pain processing in fibromyalgia. Arthritis Rheum 2002; 46: 1333-1343.

21 Vaeroy H, Helle R, Forre O, Kåss E, Terenius L. Elevated CSF levels of substance $\mathrm{P}$ and high incidence of Raynaud phenomenon in patients with fibromyalgia: new features for diagnosis. Pain 1988; 32: 21-26.

22 Russell IJ, Orr MD, Littman B, Vipraio GA, Alboukrek D, Michalek JE et al. Elevated cerebrospinal fluid levels of substance $\mathrm{P}$ in patients with the fibromyalgia syndrome. Arthritis Rheum 1994; 37: 1593-1601.

23 Shtein RM, Harper DE, Pallazola V, Harte SE, Hussain M, Sugar A et al. Discordant dry eye disease. Trans Am Ophthalmol Soc 2016; 114:(T4)[1-28].

24 Oudejans L, He X, Niesters M, Dahan A, Brines M, van Velzen $\mathrm{M}$ et al. Cornea nerve fiber quantification and construction of phenotypes in patients with fibromyalgia. Sci Rep 2016; 6: 23573.

25 Ramirez M, Martinez-Martinez LA, Hernandez-Quintela E, Velazco-Casapía J, Vargas A, Martínez-Lavín M. Small fiber neuropathy in women with fibromyalgia. An in vivo assessment using corneal confocal bio-microscopy. Semin Arthritis Rheum 2015; 45(2): 214-219.

26 Gallar J, Morales C, Freire V, Acosta MC, Belmonte C, Duran JA. Decreased corneal sensitivity and tear production in fibromyalgia. Invest Ophthalmol Vis Sci 2009; 50(9): 4129-4134.

27 Brewitt H, Sistani F. Dry eye disease: the scale of the problem. Surv Ophthalmol 2001; 45(Suppl 2): S199-S202. 